Hematolymphoid Malignancy

\title{
Plasmablastic Lymphoma: A Clinicopathological Study from a Tertiary Care Cancer Center in South India
}

\author{
Manasi C. Mundada ${ }^{1}$ Faiq Ahmed ${ }^{1}$ Rachna Khera ${ }^{1}$ \\ A. Santa² Krishnamohan Mallavarapu²
${ }^{1}$ Department of Laboratory Medicine, Basavatarakam Indo American Cancer Hospital and Research Institute, Hyderabad, Telangana, India
${ }^{2}$ Department of Medical Oncology, Basavatarakam Indo American Cancer Hospital and Research Institute, Hyderabad, Telangana, India

South Asian J Cancer:2020;9:105-108

\author{
Sudha Murthy ${ }^{1}$ Senthil Rajappa ${ }^{2}$
}

\author{
Address for correspondence Manasi C. Mundada, MD, DNB \\ (Pathology), Department of Laboratory Medicine, Basavatarakam \\ Indo American Cancer Hospital and Research Institute, Road No 10, \\ Banjara Hills, Hyderabad 500034, Telangana, India \\ (e-mail: manasicmundada@gmail.com).
}

\begin{abstract}
Keywords

- extranodal

- human immunodeficiency virus association

- immunohistochemistry

- plasmablastic lymphoma
\end{abstract}

Background Plasmablastic lymphoma (PBL) is a rare aggressive B cell lymphoma that is commonly encountered in patients with human immunodeficiency virus (HIV)/ acquired immunodeficiency syndrome (AIDS). In this case series, we describe the clinicopathological features of cases of PBL seen at a tertiary care center in South India.

Materials and Methods Medical records of patients diagnosed with PBL between January 2009 and November 2017 were reviewed. PBL was defined as per the World Health Organization 2016 classification for hematopoietic and lymphoid neoplasms. The slides were reviewed with hematoxylin and eosin along with immunohistochemistry (IHC) including CD45, CD20, PAX5, CD79a, CD3, CD5, CD138, MUMI, EMA, ALK, and Ki67. Epstein-Barr virus (EBV) association was documented by rapid in situ hybridization (RISH) studies wherever possible. The demographic data, clinical presentation, treatment details, and outcomes are elaborated using descriptive statistics.

Results During the study period, nine patients with PBL were identified. The median age at presentation was 47 years (range: $36-54$ years). All patients had associated HIV/ AIDS, eight (89\%) had extranodal disease, and six (66\%) had advanced clinical stage (stage III). All biopsies were positive for CD45, CD138, and MUM1, and negative for CD79a and T cell markers with a high Ki67 proliferation index (85-90\%); CD20 was faint positive in one patient, and CD56 was positive in one (11\%) patient. EBV-RISH was tested in two patients and was positive in one. Bone marrow was uninvolved in all the cases. At the time of last follow-up, three patients were alive. Treatment details were available in six patients. With frontline therapy, four patients achieved a complete remission (CR) and one patient developed progressive disease. Three of four patients in $C R$ are alive till the last follow-up.

Conclusion PBL is a rare form of lymphoma with predominant association with HIV, extranodal location, and characteristic IHC pattern.
DOI https://doi.org/ 10.1055/s-0040-1721194 ISSN 2278-330X. (c) 2020. Medlntel Services Pvt Ltd.

This is an open access article published by Thieme under the terms of the Creative Commons Attribution-NonDerivative-NonCommercial-License, permitting copying and reproduction so long as the original work is given appropriate credit. Contents may not be used for commercial purposes, or adapted, remixed, transformed or built upon. (https://creativecommons.org/licenses/by-nc-nd/4.0/)

Thieme Medical and Scientific Publishers Pvt. Ltd., A-12, 2nd Floor, Sector 2, Noida-201301 UP, India 


\section{Introduction}

Plasmablastic lymphoma (PBL) is a rare aggressive B cell non-Hodgkin lymphoma that is commonly associated with human immunodeficiency virus (HIV) positive individuals and is now increasingly being noted in patients without concomitant HIV infection ${ }^{1}$ PBL is characterized by loss of $B$ cell antigens, such as CD20 and PAX5, and gain of plasma cell immunophenotypes, such as CD38, CD138, and MUM1/IRF1. ${ }^{2}$ There is a strong association of PBL with Epstein-Barr virus (EBV), which is described to be positive in $75 \%$ of HIV-associated cases. ${ }^{3}$ Most of these lymphomas present in stage III / IV and are associated with poor clinical outcome.

It is important to identify this entity and differentiate it from other lesions showing plasmacytoid morphologies such as multiple myeloma, ALK + DLBCL, and large B cell lymphoma arising in HHV-8-associated multicentric Castleman's disease due to its aggressive clinical course and therapeutic implications.

There are many isolated case reports of PBL from India. However, only a few case series have been described from India. ${ }^{4-7}$ The rarity of this disease makes it difficult to conduct a randomized study at a single center; the objective of this case series is to describe the clinicopathological characteristics of PBL from India to contribute to better understanding of the clinical presentation and outcomes of this malignancy in the Indian population.

\section{Materials and Methods}

Medical records of patients diagnosed with PBL between January 2009 and November 2017 were reviewed. The demographic data, site of presentation, presence of B symptoms, nodal/extranodal involvement, clinical stage at diagnosis, serology, and CD4/ CD8 levels were noted. The biopsy slides were reviewed with hematoxylin and eosin (H\&E) and immunohistochemistry (IHC) stains including CD45 (DAKO-2B11+PD7/26), CD20 (DAKO-L26), PAX5 (DAKO-24), CD79a (DAKO-HM47/A9), CD3 (DAKO-MRQ-39), CD5 (DAKO-SP19), CD138 (DAKO-B-A38), MUM1 (DAKO-MRQ43), Ki67 (DAKO-MIB1), EMA (BIOCARE-E29), and ALK1 (BIOCARE-5A4). EBV association was documented by rapid in situ hybridization (RISH) studies wherever possible. The histological diagnosis was based on H\&E and IHC results as per the 2016 World Health Organization (WHO) classification of tumors of hematopoietic and lymphoid tissues. ${ }^{1}$ Plasmablasts were defined as large monomorphic cells with high nuclear-cytoplasmic ratio, moderate amount of amphophilic cytoplasm, and round nucleus with prominent central nucleolus. Bone marrow aspiration and biopsy were performed in all patients as a part of staging. Serum electrophoresis and light chain test were performed. A response assessment of positron emission tomography-computed tomography (CT) was performed after 3 months of initiation of treatment.

\section{Results}

There were a total of nine patients in the study. The median age of presentation was 47 years (range: $36-54$ years).
Extranodal involvement was the most frequent presentation noted in $89 \%$ (8/9) patients. The common sites of extranodal involvement included oral cavity (3), rectum (2), nasal cavity (1), and pelvic mass/iliac fossa mass (2). One patient presented with primary nodal disease. All patients were seropositive for HIV infection. In eight patients, HIV seropositivity preceded the diagnosis of PBL, whereas one patient was diagnosed with HIV seropositivity and PBL concurrently. CD4 counts at presentation were available in five patients with a median count of $115 / \mu \mathrm{L}$ (normal: $500-1,500 / \mu \mathrm{L}$ ), with three patients having a count below $200 / \mu \mathrm{L}$. Six (66\%) out of nine patients presented in stage III and two (22\%) and one (11\%) presented in stages II and I, respectively. Peripheral blood findings were within normal limits, and bone marrow was uninvolved in all the cases as ascertained on aspiration and biopsy samples. Serum electrophoresis and serum light chain results were negative in the cases studied.

On histopathological examination, patients presenting with primarily oral lesion showed plasmablastic morphology, whereas all others showed sheets of plasmablasts admixed with cells showing plasmacytic differentiation ( - Fig. 1a, b). Positive expression of CD45, CD138, and MUM1 was noted by IHC in all patients, whereas CD79a, ALK1, and T cell markers were negative on IHC staining. Faint CD20 positive was seen in one patient, whereas CD56 was positive in only one patient (with a nasal cavity mass). The Ki67 proliferation index was high (85-90\%) in all the cases (-Fig. 2a-d). EBV

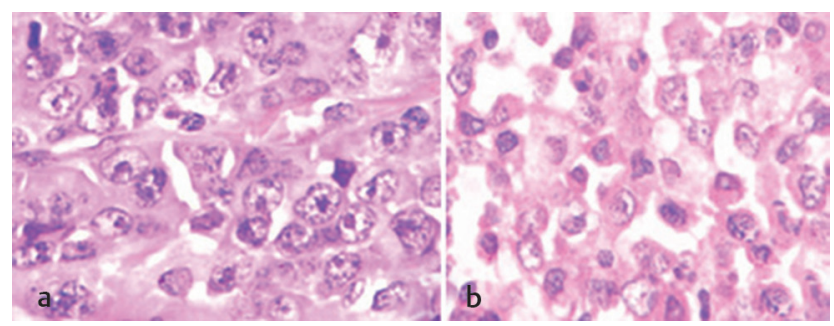

Fig. 1 (a) Plasmablastic morphology (hematoxylin and eosin, $\times 40$ ). (b) Plasmacytic cells with scattered plasmablasts (hematoxylin and eosin, $\times 40$ ).

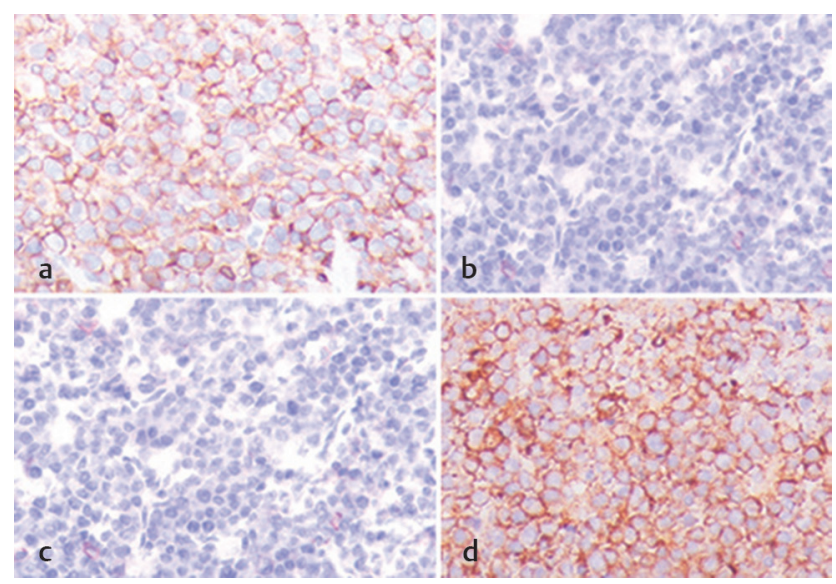

Fig. 2 (a) Leukocyte common antigen faint membrane positive in neoplastic cells ( $\times 40)$. (b) CD20 negative in neoplastic cells $(\times 40)$. (c) CD3 negative in neoplastic cells $(\times 40)$. (d) CD138 membrane positive in neoplastic cells $(\times 40)$. 


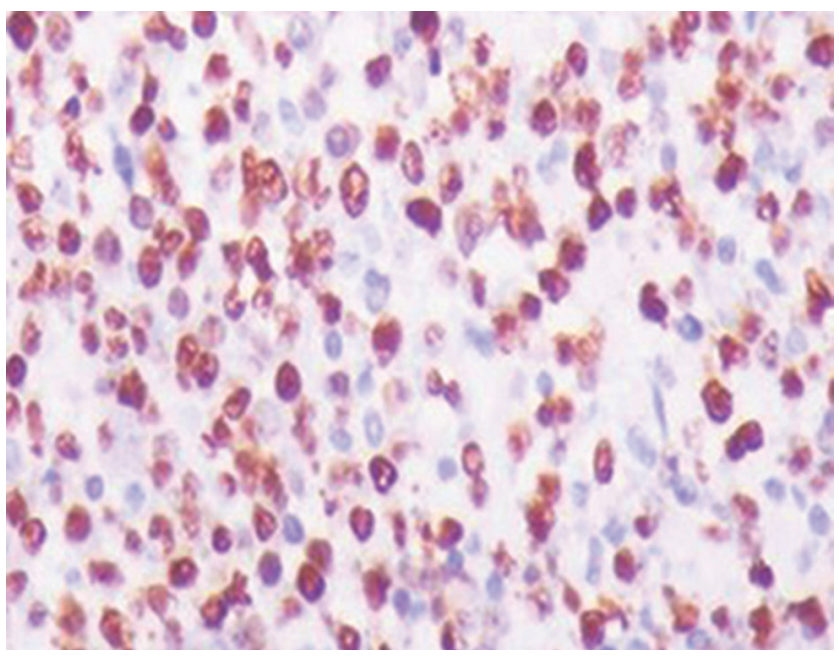

Fig. 3 Epstein-Barr virus by rapid in situ hybridization is strong nuclear positive in majority of the cells $(\times 40)$.

testing by RISH was performed in two patients, and it was

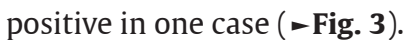

Treatment details were available in six out of nine patients. Two patients received CHOP (cyclophosphamide, vincristine, doxorubicin, and prednisone), three patients received EPOCH (infusional etoposide, vincristine, and doxorubicin with bolus cyclophosphamide and prednisone) regimens, and one patient received rituximab-EPOCH as first-line therapy. All patients received highly active antiretroviral treatment (HAART) triple-drug therapy for HIV infection as per the NACS guidelines. Of the six patients who were treated at our center, four (66\%) achieved complete remission (CR) with frontline therapy. One patient defaulted therapy after four cycles of chemotherapy, whereas one patient developed progressive disease on frontline therapy (CHOP) and was changed over to salvage therapy with dose-adjusted EPOCH. This patient died of disease progression after seven cycles of chemotherapy. Of the four patients who achieved near-total regression, one relapsed after 11 months with mesenteric deposits and was started on salvage therapy with DHAP (dexamethasone, high-dose cytarabine, and cisplatin). The other three patients with a CR remained in sustained remission at the time of last follow-up.

\section{Discussion}

PBL is a well-defined entity in the WHO 2016 classification and constitutes $2 \%$ of HIV-related lymphoma. ${ }^{3}$ However, PBL is being increasingly described in patients having immunocompromised states other than HIV. PBL commonly presents with extranodal disease, with oral cavity being the most common site. Other extranodal sites include gastrointestinal tract, nasal cavity, genitourinary tract, central nervous system, skin, lung, liver, and orbit, among others. ${ }^{1,3}$ Nodal involvement is described in $<10 \%$ of the cases. ${ }^{1}$ The postulated cell of origin for PBL is a terminally differentiated B cell, which shows a plasma cell immunophenotype. ${ }^{3}$ Given the rarity of the disease, there are limited data on the presentations and outcomes of this entity from India. The present case series of nine patients with PBL noted a concomitant HIV seropositivity in all the patients. The median age at presentation and male predominance were comparable to the western data. ${ }^{3,7,8}$ Hansra et $\mathrm{al}^{8}$ demonstrated an association of extraoral PBL with non-HIV causes of immunosuppression, which is contradictory in our study where all the patients were HIV-positive and yet 66\% showed extraoral involvement. Another study in Indian patients with PBL by Bishnu et $\mathrm{al}^{6}$ has reported similar findings. This change in the clinical presentation of disease has been attributed to better availability of HAART therapy in the recent years. ${ }^{3}$ Median CD4 count at presentation in our study was $115 / \mu \mathrm{L}$. Different studies have published varying range of CD4 counts at presentation (13-671 cells/ $\mu \mathrm{L}){ }^{8,9}$ Cattaneo et $\mathrm{al}^{9}$ have demonstrated CD4 counts $>200 / \mu$ l to be associated with a favorable prognosis in PBL. Majority (66\%) patients in our study presented with a stage III disease, a finding concordant with the literature present in western population. ${ }^{3}$ None of the patients in our study had bone marrow involvement.

Plasmablastic morphology was seen in $66 \%$ patients with an oral site of PBL in our study, which was similar to earlier observations. ${ }^{8,10}$ IHC studies showed positive expression for CD45 in all, which was weak to patchy in distribution. Plasmablastic phenotype requires the loss of $B$ cell with the gain of markers of plasmacytic differentiation. ${ }^{1}$ CD138 and MUM1 were positive in all the cases, thus confirming plasmacytic differentiation of the cells. Loss of CD79a was seen in all the cases. One of the cases showed dim CD20 expression. CD20 expression was rarely reported in the literature in PBL.,11 Expression of CD56 was unusual in PBL. Only one of nine patients in our study showed positivity. Variable positivity for CD56 was reported in PBL, and it was attributed to terminal B cell immunophenotype. ${ }^{8,12}$ The immunophenotypic characteristics were comparable in both western and Indian literature.

EBV testing by RISH was positive in one of two patients tested. The prognostic significance of EBV in HIV-positive patients is unclear; however, EBV positivity in immunocompetent patients heralds a better prognosis. ${ }^{3}$ In this study, EBV could not be tested in all the patients due to financial constraints. Furthermore, MYC translocation is also commonly known to be associated with this entity and is a poor prognostic marker for overall survival. However, data for MYC translocation were unavailable in our study.

CR was achieved in $66 \%$ of the patients at the end of first-line therapy in our study. This is fairly comparable to the CR rate of 55\% reported by Tchernonog et al in a large study of 135 patients with PBL. ${ }^{13}$ The median overall survival was 32 months in this study. Bishnu et al ${ }^{6}$ also reported similar CR rates from India. ${ }^{7}$

A comprehensive IHC panel is imperative for a definitive diagnosis of PBL. This can be particularly challenging in a resource-limited setting. A thorough clinical history and laboratory work-up to effectively rule out the more common differential diagnoses such as multiple myeloma are important as the therapies and outcomes differ drastically in these malignancies. 


\section{Conclusion}

This study highlights the varied extraoral presentation of PBL and strong association with HIV. Although a small series, it adds to the currently limited literature for PBL. The diagnosis requires IHC, which might be difficult in limited resource situations. Complete clinical history, prudent approach, and judicious use of immunostains are important in establishing a diagnosis of PBL. The rarity of the disease means that randomized studies are uncommon, and single-center or large registry-based studies provide majority of data regarding the disease characteristics and outcomes in PBL. Collaboration among regional centers is highly encouraged to establish a registry or a robust database for these patients.

\section{Funding}

None.

\section{Conflict of Interest}

There are no conflicts of interest.

\section{References}

1 Campo E, Stein H, Harris NL, Plasmablastic lymphoma. In: Swerdlow SH, Campo E, Harris NL, et al, eds. WHO Classification of Tumours of Haematopoietic and Lymphoid Tissues. Revised 4th ed. Lyon, France: IARC Press; 2017 321-322

2 Delecluse HJ, Anagnostopoulos I, Dallenbach F, et al. Plasmablastic lymphomas of the oral cavity: a new entity associated with the human immunodeficiency virus infection. Blood 1997;89(4):1413-1420

3 Castillo JJ, Bibas M, Miranda RN. The biology and treatment of plasmablastic lymphoma. Blood 2015;125(15):2323-2330
4 Komaranchath AS, Haleshappa RA, Kuntegowdenahalli LC, Kumar RV, Dasappa L, Babu G. Plasmablastic lymphoma of the gastrointestinal tract: A rare entity with a dismal prognosis. Indian J Cancer 2016;53(4):529-533

5 Reddy R, Gogia A, Kumar L, et al. HIV-associated hematologic malignancies: experience from a tertiary cancer center in India. Indian J Med Paediatr Oncol 2016;37(3):141-145

6 Bishnu S, Banerjee S, Bandyopadhyay D, Samui S, Bhattacharya S, Bose D. Plasmablastic lymphoma in HIV patients: Experience at a tertiary care hospital in eastern India. Indian J Cancer 2015; 52(4):563-567

7 Babu KG, Suresh Babu MC, Abraham LJ, et al. Plasmablastic lymphoma: does prognosis differ with HIV status and site of disease? Oncol Gastroenterol Hepatol Rep 2014;3:25-29

8 Hansra D, Montague N, Stefanovic A, et al. Oral and extraoral plasmablastic lymphoma: similarities and differences in clinicopathologic characteristics. Am J Clin Pathol 2010; 134(5):710-719

9 Cattaneo C, Re A, Ungari M, et al. Plasmablastic lymphoma among human immunodeficiency virus-positive patients: results of a single center's experience. Leuk Lymphoma 2015;56(1):267-269

10 Gujral S, Shet TM, Kane SV. Morphological spectrum of AIDSrelated plasmablastic lymphomas. Indian J Pathol Microbiol 2008;51(1):121-124

11 Montes-Moreno S, Gonzalez-Medina AR, Rodriguez-Pinilla SM, et al. Aggressive large B-cell lymphoma with plasma cell differentiation: immunohistochemical characterization of plasmablastic lymphoma and diffuse large B-cell lymphoma with partial plasmablastic phenotype. Haematologica 2010;95(8):1342-1349

12 Vega F, Chang CC, Medeiros LJ, et al. Plasmablastic lymphomas and plasmablastic plasma cell myelomas have nearly identical immunophenotypic profiles. Mod Pathol 2005;18(6):806-815

13 Tchernonog E, Faurie P, Coppo P, et al. Clinical characteristics and prognostic factors of plasmablastic lymphoma patients: analysis of 135 patients from the LYSA group. Ann Oncol 2017; 28(4):843-848 